\title{
AVALIAÇÃO DA PRESENÇA DE FORMALDEÍDO EM PRODUTOS CAPILARES
}

\section{EVALUTION OF THE PRESENCE OF FOMALDEHYDE IN CAPILLARY PRODUCTS}

Francielly da Silva Morais Ferreira ${ }^{1}$, Maíra Rodrigues Uliana ${ }^{1}$, Daniel Ângelo Macena ${ }^{2}$, Vinicius Marques Gomes ${ }^{1}$

${ }^{1}$ Universidade do Oeste Paulista - UNOESTE. ${ }^{2}$ Universidade Virtual do Estado de São Paulo-UNIVESP.

E-mail: francieellyferreira@hotmail.com

RESUMO - O Formaldeído é usado em algumas formulações de produtos cosméticos, mas segundo a ANVISA, somente pode ser utilizado como agente conservante com limite máximo de $0,2 \%$ e como endurecedor de unha com limite de $5 \%$. Apesar da ANVISA determinar o limite máximo permitido de formaldeído, algumas marcas de produtos fazem o mascaramento do odor característico do formaldeído. O presente estudo analisou três marcas de alisantes e tinturas capilares através do método de espectrofotometria. Onde evidenciou a presença de formaldeído em todas as amostras, resultando dessa maneira em valores que variam de $1,6 \%$ a $7 \%$ de concentração acima do permitido pela ANVISA (2013). Os fios de cabelo possui um $\mathrm{pH}$ que varia de 4,5 a 5,5. As amostras A5 e A6 foram compatíveis com as características fisiológicas do cabelo não agredindo a estrutura interna do fio, enquanto as amostras A1, A2, A3, A4, B1,B2, B3, B4,B6 e B7 estavam com o pH fora da faixa o que pode causar danificação da estrutura do fio de cabelo. Nenhuma das amostras analisadas foi possível sentir o odor do formaldeído. Dessa maneira existe uma necessidade de um maior acompanhamento e vigilância a cerca dos produtos cosméticos, principalmente as tintas capilares que resultaram em concentrações elevadíssimas de formaldeído que possui uma alta toxicidade e causas sintomas prejudicais de imediato ou em longo prazo a saúde humana.

Palavras-chave: Espectrometria; Saúde; Formaldeído.

ABSTRACT - Formaldehyde is used in some cosmetic formulations, but according to ANVISA it can only be used as a preservative agent with a maximum limit of $0.2 \%$ and as a nail hardener with a limit of $5 \%$. Although ANVISA determines the maximum allowable limit of formaldehyde, some product brands mask the characteristic odor of formaldehyde. The present study analyzed three brands of hair straighteners and dyes using the spectrophotometry method. Where evidenced the presence of formaldehyde in all samples, thus resulting in values ranging from $1.6 \%$ to $7 \%$ concentration above that allowed by ANVISA (2013). Hair strands have a $\mathrm{pH}$ ranging from 4.5 to 5.5. Samples A5 and A6 were compatible with the physiological characteristics of hair not affecting the internal structure of the hair, while samples $A 1, A 2, A 3, A 4, B 1, B 2, B 3, B 4, B 6$ and $B 7$ had $\mathrm{pH}$ out of range. which can cause damage to the hair structure. None of the samples analyzed could 
smell formaldehyde. Thus there is a need for greater monitoring and surveillance around cosmetic products, especially hair dyes that have resulted in very high concentrations of formaldehyde that has a high toxicity and causes immediate or long-term harmful symptoms to human health.

Keywords: Spectrometry; Health; Formaldehyde. 


\section{INTRODUÇÃO}

Cabelo é uma junção de várias ligações peptídica na formação de um polímero biológico. É através desse polímero que o cabelo possui suas características de cor, comprimento, densidade, peso e formatado, que vai desde cabelo liso ao crespo. Possui várias ligações químicas importantes tanto nas transformações no processo de mudança do cabelo, como alisamentos e tingimento, e também na manutenção e estrutura do cabelo (BREMER;HEPP, 2012; OLIVEIRA, 2013).

Um dos processos mais comuns é o tingimento do cabelo. Esse processo é caracterizado pela mudança de cor através de várias técnicas utilizadas ao longo dos anos. A forma mais usada atualmente são as colorações permanentes. Existem também as colorações semipermanentes e temporárias (KIRKLAND et al., 2005).

Tintas semipermanentes e temporárias são depositadas nos fios do cabelo, preferencialmente utilizada em pessoas no processo de retocar a raiz dos fios. Normalmente são retocadas a cada 15 a 30 dias por serem eliminadas após algumas lavagens e são menos agressivas aos fios de cabelo (OLIVEIRA, 2014).

Tintas permanentes precisam de uma abertura nos fios e a absorção é feita através do córtex. Nessas tinturas, é utilizado o peroxido de hidrogênio, que oxidam as substancias intermediarias se ligando a acopladores produzindo a cor desejada. A solução amoniacal de $\mathrm{pH} 8$ a $10 \mathrm{faz}$ a abertura das cutículas do fio para absorção tanto do peroxido de hidrogênio quanto do pigmento da cor desejada (PINHEIRO, 2008).

Outro processo muito utilizando mundialmente é o alisamento capilar, que promete bom resultado ao longo de aproximadamente quatro meses e sua aplicação cresce a cada ano (BALOCH et al., 2009).

Estes produtos possuem em suas formulações várias substancias, dentre elas o formaldeído, que segundo a ANVISA em sua resolução $n^{\circ}$ 162, de 2001 não pode ultrapassar 0,2 \% da composição do produto e é empregada como agente conservante. Existem também outras substancias que tem como função alterar a estrutura do cabelo como glutaraldeído, hidróxido de sódio, hidróxido de cálcio, pirogalhol e ácido tioglicólico presente nesses produtos (PINHEIRO, 2009).

O formaldeído, também como metanal, é uma substancia do grupo etílico, sendo o aldeído mais simples do grupo, com apenas um carbono e obtido através da oxidação do metanol, um álcool primário. É facilmente encontrado na natureza em alimentos, é incolor, apresenta odor característico, em sua forma gasosa é altamente inflamável e muito utilizado em produtos cosméticos em seu estado aquoso. Esta solução aquosa de formaldeído a 35$50 \%$ esta presente o metanol como um agente preservativo contra a polimerização, popularmente conhecido como formol (MACAGNAN, SARTORI; CASTRO, 2010).

Esses produtos, mesmo com avanço das tecnologias para diminuir o risco a saúde, são muito consumidos devido a vários fatores, como rótulos ilegíveis e de difícil compreensão e a manipulação dos consumidores por parte dos fabricantes ocultando a presença das substâncias. E com esse objetivo que a Agencia Nacional de Vigilância Sanitária (ANVISA) criou uma resolução RDC N 03, de 20 janeiro de 2012 para valores máximos permitidos para cada substancia (GOMES, 1999).

Ainda assim, no Brasil, existe um fraco monitoramento destes produtos de cosméticos, que pode possui uma alta quantidade de determinada substancia e também $o$ surgimento de marcas clandestinas que pode trazer riscos à saúde (PINHEIRO, 2009).

Desde modo, é necessário informar aos consumidores, de uma maneira clara, os riscos envolvidos na utilização de tais produtos através de rótulos legíveis $e$ políticas de divulgação. Nesse sentido de verificação a presença de formaldeído nesses cosméticos, a utilização do método de 
espectrofometria terá a capacidade de avaliar a concentração de formaldeído em cremes de alisamento e tinturas capilares e relacionar com as informações contidas nos rótulos dos fabricantes, como o exigido pela resolução da ANVISA nos anexos IV e $\mathrm{V}$ da RDC 211/2005.

\section{MÉTODO}

\subsection{Aquisições das amostras}

Para o desenvolvimento da pesquisa, foram adquiridas dois lotes de três diferentes fabricantes de creme alisantes e de tinturas capilares devidamente lacradas. As amostras de creme alisantes serão classificadas como (A) e as de tinturas capilares como (B). O procedimento foi realizado em triplicata com finalidade de garantir exatidão nos resultados.

\subsection{Reagentes}

Para a realização da pesquisa serão utilizados os seguintes reagentes: formaldeído PA 37\%, ácido cromotrópico 99\% (sal dissódicodilhidratado), sulfato de magnésio $98 \%$, todos de grau analítico.

\subsection{Equipamentos utilizados no experimento} Espectrofotômetro, banho termostatizado, pHmetro, agitador magnético e balança analítica.

\subsection{Curva analítica de identificação e determinação da concentração do formaldeído. \\ Identificou-se a presença de} formaldeído nas soluções através do composto colorido que é formado devido à reação que ocorre entre o ácido cromotrópico e o formaldeído na presença de sulfato de magnésio após aquecimento.

Para determinação da curva do formaldeído, foram realizados o seguinte ensaio : 90; 150 e $230 \mu \mathrm{L}$ da solução estoque contendo formaldeído foram transferidas para tubos de ensaio. Em seguida, foram adicionados aos tubos $290 \mu \mathrm{L}$ de solução de ácido cromotrópico a $5 \%(\mathrm{p} / \mathrm{v})$ e $3,0 \mathrm{~mL}$ de solução de sulfato de magnésio a $60 \%(\mathrm{p} / \mathrm{v})$, com agitação. Os tubos foram aquecidos durante 60 minutos em banho de vapor $\left(100^{\circ} \mathrm{C}\right)$, seguido do esfriamento ate $25^{\circ} \mathrm{C}$. As soluções foram transferidas para balões de $25 \mathrm{~mL}$ e o volume completado com água destilada, obtendo as seguintes concentrações de formaldeído (ppm): 3,6; 6,0 e 9,2. As medições da absorbância foram registradas a $535 \mathrm{~nm}$. Todas as analises foram feitas em triplicata para garantir a exatidão dos resultados obtidos.

\subsection{Preparo de soluções}

A metodologia utilizada para identifica e quantificar o teor de formaldeído nas amostras será aplicada de acordo com o método padrão recomendado pelo Instituto Nacional de Segurança e Saúde Ocupacional (NIOSH, 1994), seguido das modificações descritas por Gasparini (Gasparini et al., 2008). O formaldeído a 37\% (v/v) diluído com água destilada em um balão volumétrico de $1000 \mathrm{~mL}$. A solução aquosa de ácido cromotrópico (CA) a $5 \%(\mathrm{p} / \mathrm{v})$ será preparada dissolvendo-se $1,25 \mathrm{~g}$ de soluto em água destilada em balão volumétrico de $25 \mathrm{~mL}$. Já a solução aquosa de sulfato de magnésio (MgSO4.7H2O) a 60\% (p/v), dissolvendo $60 \mathrm{~g}$ do soluto em água destilada em um balão volumétrico de $100 \mathrm{~mL}$.

\subsection{Preparo da amostra e determinação de formaldeído}

Pesou-se 3,0 g de cada amostra comercial, em seguida dissolvida em cerca de $20 \mathrm{~mL}$ de água destilada agitando por aproximadamente 5 minutos em agitador magnético e completado ate seu volume final de $100 \mathrm{~mL}$, obtendo dessa maneira uma solução de concentração (C1) de $30.000 \mu$ g. $\mathrm{mL}-1$. Em seguida $5,0 \mathrm{~mL}$ dessa solução (C1) diluída em $25 \mathrm{~mL}$ de água destilada obtendo uma concentração de $6.000 \mu \mathrm{g}$. $\mathrm{mL}-1$. Uma alíquota de $1,0 \mathrm{~mL}$ da solução (C2) foram transferidas para um tubo de ensaio juntamente com $290 \mu \mathrm{L}$ de ácido cromotrópico $5 \%(p / v)$ e 3,0 $\mathrm{mL}$ de sulfato de magnésio a $60 \%(\mathrm{p} / \mathrm{v})$. Em seguida, levado ao aquecimento em banho de vapor a $100{ }^{\circ} \mathrm{C}$ 
durante 60 minutos. A solução resultante transferiu-se para um balão volumétrico de $25 \mathrm{~mL}$ completando o volume com água destilada resultando em uma concentração (C3) de $240 \mu \mathrm{g}$. mL-1. Essas soluções foram medidas no espectrômetro a $535 \mathrm{~nm}$.

\subsection{Características organolépticas e físico- químicas}

Foram realizados ensaios organolépticos e físicos- químicos para apreciar as características dos produtos em estudo. As características avaliadas serão: odor, cor, aspecto, $\mathrm{pH}$ e análise de rotulo (BRASIL, 2008; ANVISA, 2005).

\subsection{Determinação do $\mathrm{pH}$}

A determinação do $\mathrm{pH}$ foram realizadas através do método potenciométrico. Realizou-se a diluição das amostras a $10 \%(\mathrm{p} / \mathrm{v})$ em agua destilada, temperatura ambiente, em triplicata. (Brasil,2008;Isaac et al., 2008; Farmacopeia Brasileira, 2010.)

\subsection{Análise de rótulo}

As analises foram feitas seguindo os critérios estabelecidos pelas legislações nacionais - RDC 211/2005 que define as normas de rotulagem obrigatórias para produtos cosméticos e RDC 332/2005 que trata da regulamentação e implantação da cosmetovigilância nas indústrias de cosméticos. Foi realizada de uma maneira visual crítica e qualitativa nos rótulos de cada fabricante.

\section{RESULTADOS}

Curva analítica de determinação e identificação do formaldeído

A determinação da presença de formaldeído foi evidenciada através da produção de um composto marrom avermelhado resultante da reação que ocorre entre o formaldeído com ácido cromotrópico na presença de sulfato de magnésio mediante a aquecimento.

A curva analítica apresenta uma regressão linear significativa, cuja equação da reta é dada: $A=0,0224 \times$ (solução de formaldeído, ácido cromotrópico e sulfato de magnésio ) (ppm) +0,0001. O coeficiente de correlação obtido foi de 0,9981.

Todas as amostras desenvolveram um composto de coloração marrom avermelhada após aquecimento, indicando a presença de formaldeído nas diferentes amostras de alisantes capilares e tintas, evidenciando que quanto maior a concentração mais escura tende de ficar a amostra. A amostra que evidenciou uma coloração mais intensa as demais foi a B1, justiçando dessa maneira uma concentração maior de formaldeído. A amostra que evidenciou menor coloração das demais foi a $A 3$, comprovando dessa maneira uma menor concentração de formaldeído. As amostras A1, A2, A4, A5, A6, B2, B3, B4, $\mathrm{B} 5$ e $\mathrm{B} 6$ evidenciam intensidades variadas de coloração e concentração. Os percentuais de formaldeído calculados através da equação da reta proveniente da curva analítica confirmam os resultados e estão na descritos na figura 1.

Características organolépticas e físicoquímicas

As amostras em estudo obtiveram os resultados organolépticos e físico-químicos e estão relacionados na tabela 1.

As analise de aspecto e cor, foram determinadas observando visualmente, as amostras apresentaram cremes e tintas homogenias e colorações variadas. O odor foi aferido diretamente através do olfato, nenhuma das amostras analisadas possui o odor característico do formaldeído conseguindo mascarar a presença da substancia no produto. As amostras A1, A2, A3, A4 apresentam odor de enxofre intenso característico do tioglicolato de amônia. As amostras A5, A6, B1, B2 não apresentaram nenhum odor irritante ou forte, apenas de suas essências usadas nesses produtos, dessa maneira mascarando a presença do formaldeído nas amostras. As amostras B3, B4, B5, B6 apresentaram um odor amoniacal forte e um pouco irritante. 
Figura 1. Representação gráfica do porcentual de formaldeído nas amostras A1A6 e B1- B6.

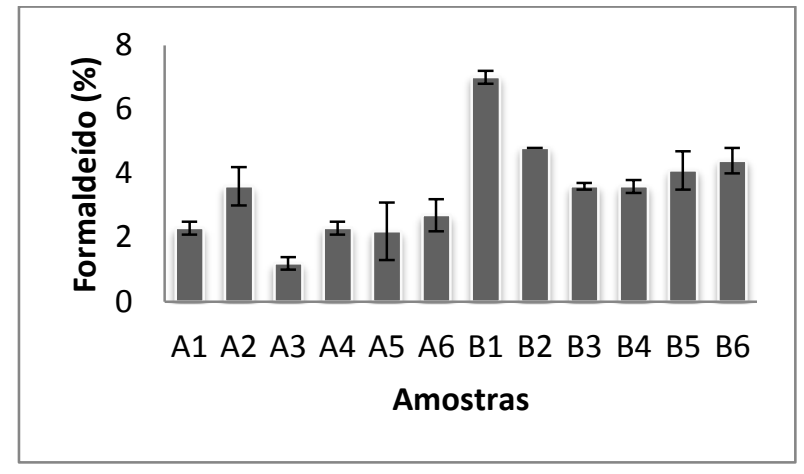

As amostras A5, A6 apresentaram um $\mathrm{pH}$ levemente ácido, que são capazes de fechar a cutícula capilar. As amostras A1, A2, A3, A4, B1, B2, B3 apresentam pH extremamente elevado que são predominantes de cremes capilares e tintas de capilares, que abrem a cutícula do cabelo permitindo dessa maneira que o produto entre na estrutura interna do cabelo.

Tabela 1. Características organolépticas e físico-químicas das amostras de creme alisantes e tintas capilares.

\begin{tabular}{cccccccc}
\hline ENSAIOS & A1 & \multicolumn{2}{c}{ A2 } & A3 & A4 & A5 & A6 \\
\hline Aspecto & CH & CH & CH & CH & CH & CH \\
Cor & BR & BR & BR & BR & PR & PR \\
Odor & E & E & E & E & S & S \\
pH & $9,08 \pm$ & $9,1 \pm$ & $9,01 \pm$ & $8,96 \pm$ & $3,16 \pm$ & $3,17 \pm$ \\
& 0,1 & 0 & 0 & 0,4 & 0,1 & 0 \\
ENSAIOS & B1 & B2 & B3 & B4 & B5 & B6 \\
Aspecto & CH & CH & CH & CH & CH & CH \\
Cor & AM & AM & AM & AM & CA & AM \\
Odor & A & A & S & S & A & A \\
pH & $10,23 \pm$ & $9,67 \pm$ & $10,21 \pm$ & $10,14 \pm$ & $14 \pm$ & $14,1 \pm$ \\
& 0,5 & 1,3 & 0 & 0 & 0 & 0,1 \\
\hline
\end{tabular}

Legenda: $\mathrm{CH}$ - Creme Homogêneo; E - Enxofre (tioglicolato de amônia); S - Sem odor característico; ;A - Amoniacal; BR - Branca; PR - Preta; AM - Amarelada; CS - Castanha.

\section{Análise do rotulo}

Os resultados das amostras analisadas no presente estudo estão representados na tabela 2 e 2.1. Todas as amostras não indicam a presença de formaldeído em seus rótulos. As amostras A3, A4, A5, A6, B4 e B5 possuem outras irregularidades no rótulo, ignorando dessa maneira as informações de manter fora do alcance das crianças. As amostras A5, A6, B1, B2, B3, B4, B5, B6 apresentam outra irregularidade no rótulo que deve indicar que o preparo somente deve ser usado para o fim que se destina, sendo PERIGOSO para qualquer outro uso. As amostras B3, B4, B5, B6 possui irregularidades no rótulo, essa amostras não possui registro na ANVISA ate a presente data do estudo. 
Tabela 2. Itens necessários nos rótulos dos produtos de acordo com os anexos IV e $V$ da RDC 211/2005

\begin{tabular}{lcccccc}
\hline AMOSTRAS/ AVALIAÇÃO DE ROTULO & A1 & A2 & A3 & A4 & A5 & A6 \\
\hline Nome do produto & $\mathrm{S}$ & $\mathrm{S}$ & $\mathrm{S}$ & $\mathrm{S}$ & $\mathrm{S}$ & $\mathrm{S}$ \\
Marca & $\mathrm{S}$ & $\mathrm{S}$ & $\mathrm{S}$ & $\mathrm{S}$ & $\mathrm{S}$ & $\mathrm{S}$ \\
$\mathbf{N}^{\circ}$ registro & $\mathrm{S}$ & $\mathrm{S}$ & $\mathrm{S}$ & $\mathrm{S}$ & $\mathrm{S}$ & $\mathrm{S}$ \\
Lote & $\mathrm{S}$ & $\mathrm{S}$ & $\mathrm{S}$ & $\mathrm{S}$ & $\mathrm{S}$ & $\mathrm{S}$ \\
Prazo de Validade & $\mathrm{S}$ & $\mathrm{S}$ & $\mathrm{S}$ & $\mathrm{S}$ & $\mathrm{S}$ & $\mathrm{S}$ \\
Fabricante/ Importador/ Titular & $\mathrm{S}$ & $\mathrm{S}$ & $\mathrm{S}$ & $\mathrm{S}$ & $\mathrm{S}$ & $\mathrm{S}$ \\
Pais de Origem & $\mathrm{S}$ & $\mathrm{S}$ & $\mathrm{S}$ & $\mathrm{S}$ & $\mathrm{S}$ & $\mathrm{S}$ \\
CNPJ & $\mathrm{S}$ & $\mathrm{S}$ & $\mathrm{S}$ & $\mathrm{S}$ & $\mathrm{S}$ & $\mathrm{S}$ \\
Ingredientes & $\mathrm{Fp} / \mathrm{N}$ & $\mathrm{Fp} / \mathrm{N}$ & $\mathrm{Fp} / \mathrm{N}$ & $\mathrm{Fp} / \mathrm{N}$ & $\mathrm{Fp} / \mathrm{N}$ & $\mathrm{Fp} / \mathrm{N}$ \\
Uso profissional & $\mathrm{S}$ & $\mathrm{S}$ & $\mathrm{S}$ & $\mathrm{S}$ & $\mathrm{S}$ & $\mathrm{S}$ \\
Modo de uso (se for necessário) & $\mathrm{S}$ & $\mathrm{S}$ & $\mathrm{S}$ & $\mathrm{S}$ & $\mathrm{S}$ & $\mathrm{S}$ \\
Advertências/ Restrições de uso & $\mathrm{S}$ & $\mathrm{S}$ & $\mathrm{S}$ & $\mathrm{S}$ & $\mathrm{S}$ & $\mathrm{S}$ \\
Não aplicar se o couro cabeludo estiver & $\mathrm{S}$ & $\mathrm{S}$ & $\mathrm{S}$ & $\mathrm{S}$ & $\mathrm{S}$ & $\mathrm{S}$ \\
irritado ou lesionado & & & & & & \\
Manter fora do alcance das crianças & $\mathrm{S}$ & $\mathrm{S}$ & $\mathrm{N}$ & $\mathrm{N}$ & $\mathrm{N}$ & $\mathrm{N}$ \\
& & & & & & $\mathrm{N}$ \\
Este produto somente deve ser usado & $\mathrm{S}$ & $\mathrm{S}$ & $\mathrm{S}$ & $\mathrm{S}$ & $\mathrm{N}$ \\
para o fim a que se destina, sendo & & & & & & \\
PERIGOSO para qualquer outro fim & & & & & \\
\hline
\end{tabular}

Legenda: S - Indicado no rotulo; N - Não indicado no rótulo; Fp/ N - Formaldeído presente/ Não indicado no rótulo.

Tabela 2.1. Itens necessários nos rótulos dos produtos de acordo com os anexos IV e $\mathrm{V}$ da RDC 211/2005

\begin{tabular}{|c|c|c|c|c|c|c|}
\hline AMOSTRAS/ AVALIAÇÃO DE ROTULO & B1 & B2 & B3 & B4 & B5 & B6 \\
\hline Nome do produto & $\mathrm{S}$ & $S$ & $\mathrm{~S}$ & $S$ & $\mathrm{~S}$ & $\mathrm{~S}$ \\
\hline Marca & $\mathrm{S}$ & S & S & $\mathrm{S}$ & S & $S$ \\
\hline $\mathrm{N}^{\circ}$ registro & $\mathrm{N}$ & $\mathrm{N}$ & $\mathrm{N}$ & $\mathrm{N}$ & $S$ & $S$ \\
\hline Lote & $\mathrm{S}$ & $\mathrm{S}$ & $S$ & $\mathrm{~S}$ & $S$ & $S$ \\
\hline Prazo de Validade & $\mathrm{S}$ & S & $S$ & $\mathrm{~S}$ & $S$ & $S$ \\
\hline Fabricante/ Importador/ Titular & $\mathrm{S}$ & $S$ & $\mathrm{~S}$ & $S$ & $S$ & $S$ \\
\hline Pais de Origem & $\mathrm{S}$ & S & $\mathrm{S}$ & $S$ & $\mathrm{~S}$ & $S$ \\
\hline CNPJ & $\mathrm{S}$ & $\mathrm{S}$ & $S$ & $\mathrm{~S}$ & S & $S$ \\
\hline Ingredientes & $\mathrm{Fp} / \mathrm{N}$ & $\mathrm{Fp} / \mathrm{N}$ & $\mathrm{Fp} / \mathrm{N}$ & $\mathrm{Fp} / \mathrm{N}$ & $\mathrm{Fp} / \mathrm{N}$ & $\mathrm{Fp} / \mathrm{N}$ \\
\hline Uso profissional & $\mathrm{S}$ & $S$ & $\mathrm{~S}$ & $\mathrm{~S}$ & $\mathrm{~S}$ & $S$ \\
\hline Modo de uso (se for necessário) & $\mathrm{S}$ & $S$ & $\mathrm{~S}$ & $\mathrm{~S}$ & $\mathrm{~S}$ & $S$ \\
\hline Advertências/ Restrições de uso & $\mathrm{S}$ & S & $\mathrm{S}$ & $\mathrm{S}$ & $\mathrm{S}$ & $S$ \\
\hline $\begin{array}{l}\text { Não aplicar se o couro cabeludo estiver } \\
\text { irritado ou lesionado }\end{array}$ & $\mathrm{S}$ & $\mathrm{S}$ & $\mathrm{N}$ & $\mathrm{N}$ & $\mathrm{S}$ & $S$ \\
\hline Manter fora do alcance das crianças & $\mathrm{S}$ & $\mathrm{S}$ & $S$ & $\mathrm{~S}$ & $S$ & $S$ \\
\hline $\begin{array}{l}\text { Este produto somente deve ser usado } \\
\text { para o fim a que se destina, sendo } \\
\text { PERIGOSO para qualquer outro fim }\end{array}$ & $\mathrm{N}$ & $\mathrm{N}$ & $\mathrm{N}$ & $\mathrm{N}$ & $\mathrm{N}$ & $\mathrm{N}$ \\
\hline
\end{tabular}




\section{DISCUSSÃO}

O estudo realizado evidencia que o método utilizado atende os requisitos da química verde na geração de menos resíduos tóxicos, uma vez que o método de referencia NIOSH 3500 (1994), utiliza ácido sulfúrico concentrado juntamente com ácido cromotrópico para determinação de formaldeído, que possui um alta corrosividade e toxicidade.

Segundo Toutianoush et al. (2005), a determinação do formaldeído por espectrometria se deve a presença do sulfato de magnésio e o ácido cromotrópico em excesso que após o aquecimento produz um composto avermelhado. A reação não ocorre na ausência do sulfato de magnésio. O método foi utilizado por Gasparini et al, para determinar formaldeído e desinfetantes e desfrisantes capilares comprovando que o método possui um baixo custo operacional e simples, todavia também seletivo assim como o método referencial NIOSH 3500 (1994).

O método proposto mostrou-se apropriado e com um boa aplicabilidade, possibilitando identificar a presença do formaldeído em todas as amostras, com concentrações que variam de 1,6 \% a 7\% $(p / v)$, que consisti em concentrações 8 a 35 vezes acima do permitido pela ANVISA (2013).

Outros autores utilizaram o método de analise por espectrometria e evidenciaram em seus estudos concentrações altas de formaldeído em diferentes produtos usados no procedimento de escova progressiva, variando entre 1,6 e $10,5 \%$. Produtos com alta concentração de formaldeído foram citados como percursores de sintomas como espirros, dispneia, tosse e irritação do trato respiratório por $23 \%$ dos cabeleiros pesquisados em um estudo realizado por Beloto \& Bertolin (2005). Mostrando o profundo desconhecimento e despreocupação por partes dos consumidores e cabelereiros diante dos riscos causados em longo prazo a saúde humana. As amostras de tintas capilares evidencia a falta de informação nos rótulos e fiscalização por parte da ANVISA, uma vez que o formaldeído somente pode ser usando como conservante a no máximo $0,2 \%$ em produtos cosméticos. A amostra B1 resultou em uma concentração 35 vezes maior que o permitindo e a amostra B2 do mesmo fabricante porem de lote diferente evidenciou uma concentração 24 vezes maior, mostrando uma diminuição nos diferentes lotes, mas acima ainda do permitido pela ANVISA. As amostras de tintas capilares resultaram em concentrações maiores que as dos cremes alisantes, provavelmente para conservar as mesmas por um período de tempo maior e também pela falta de uma fiscalização rigorosa sobre esses produtos. Dessa maneira os consumidores e cabeleiros não possuem conhecimento sobre a presença do formaldeído nesses produtos, uma vez que existem poucos estudos relacionados às tintas capilares em relação à determinação de formaldeído nesses produtos.

As analises de características organolépticas e físico-químicas são importantes de ser analisadas uma vez que os consumidores levam em consideração principalmente o odor dos produtos, aspecto e cor, mas alguns produtos mesmo usando essências extremamente perfumadas ainda é possível sentir o cheiro característico do formaldeído. Nenhuma das amostras analisadas foi possível sentir o odor do formaldeído, as amostras resultaram em odor característico do próprio tioglicolato, amônio e essências. A cor do produto, no entanto não possui ligação com a presença ou não de formaldeído.

Os fios de cabelo possui um $\mathrm{pH}$ que varia de 4,5 a 5,5. Produtos capilares possuem variações extremas de $\mathrm{pH}$ que podem danificar os fios de cabelo, isso porque em $\mathrm{pH}$ muito ácido aumenta a porosidade e as camadas das cutículas se delatam, obtendo dessa maneira uma aparência ressecada e opaca e pode chegar a dissolução completa do fio em $\mathrm{pH}$ fortemente alcalino. As amostras A5 e A6 
foram compatíveis com as características fisiológicas do cabelo não agredindo a estrutura interna do fio, enquanto as amostras A1, A2, A3, A4, B1, B2, B3, B4,B6 e $B 7$ estavam com o $\mathrm{pH}$ fora da faixa o que pode causar danificação da estrutura do fio de cabelo, devido a isso os produtos de uso profissional devem ser utilizados e aplicados por profissionais capacitados e utilizados com cautela.

Dessa maneira e imprescindível a avaliação dos parâmetros de qualidade dos produtos capilares, uma vez que o mercado de cosméticos é um ramo que cresce bastante no Brasil, e as formulações milagrosas que prometem um alisamento perfeito sem a presença de formaldeído, mas que podem apresentar uma concentração elevada e mascarar o odor característico do formaldeído através de essências extremadamente perfumadas e sem identificação no rotulo, assim como o presente estudo comprovou que $100 \%$ das amostras para alisamento e tingimento capilar estão com as concentrações altas e fora do permitido.

\section{CONSIDERAÇÕES FINAIS}

Através dos dados do presente estudo obtidos, evidencia dessa maneira a necessidade de um maior acompanhamento e vigilância a cerca dos produtos cosméticos, principalmente as tintas capilares que resultaram em concentrações elevadíssimas de formaldeído que possui uma alta toxicidade e causas sintomas prejudicais de imediato ou em longo prazo a saúde humana. A implantação do sistema de cosmetovigilância é de extrema importância para que os produtos possuam uma maior qualidade e informações em seus rótulos devidamente corretos, legíveis de fácil compreensão tanto na forma de uso como na composição do mesmo, gerando dessa maneira uma maior segurança ao consumidor. O sistema criado traria enormes benefícios ao setor de cosméticos com um todo. Umas das medidas de ações, seria fazer palestra mostrando a população a cerca dos estudos realizados por diversos pesquisadores do perigo que a falta de informação pode trazer a saúde humana, panfletos de orientação que possibilitara a vigilância efetiva por parte dos consumidores.

\section{REFERÊNCIAS}

ABRAHAM, L. S. et al. Tratamentos estéticos e cuidados dos cabelos: uma visão médica (parte 2). Medicina Educacional, Rio de Janeiro, v.1, n.4, nov., 2009.

ANDERSEN, I.; PROCTOR, D. F. The rate and effects of inhaled materials. In: Andersen I, editor. The nose, upper airway physiolgy and the atmospheric environment. Amsterdam: Elsevier Biomedical; 1982.p.4237.

AGÊNCIA NACIONAL DE VIGILÂNCIA SANITÁRIA. RDC no 211 de 14 de julho de 2005. Estabelece a definição e a classificação de produtos de higiene pessoal, cosméticos e perfumes, conforme Anexo I e II desta Resolução. Diário Oficial da União, Brasília, DF, 18 jul. 2005.

BALOGH, T. S. et al. Escova progressiva, abordagem crítica do uso de formaldeído em procedimentos de alisamento. São Paulo Instituto de Pesquisa e tratamento do cabelo e da pele, 2009.

BEDIN, V. Vitaminas e Produtos capilares. Cosmetics \& Toiletries: Tecnopress, São Paulo, v.18, n.4, p.50, nov./dez. 2006.

BEINADES, A. Tintes Capilares. Evolucion histórica y situación actual. OFFMAM, v.26, n.10, p. 68-72, nov. 2007.

BOLDUC, C.; SHAPIRO, J. Hair care products: waving, straightening, conditioning and coloring. Clinics in Dermatology., v.19, n.4, p.431-436, 2001. https://doi.org/10.1016/S0738081X(01)00201-2 
BRASIL. Agência Nacional de Vigilância Sanitária. Guia de controle de qualidade de produtos cosméticos: Uma abordagem sobre ensaios físicos e químicos. Brasília: Anvisa, 2007.

BRASIL. Agência Nacional de Vigilância Sanitária. Guia para avaliação de segurança de produtos cosméticos. Brasília: Anvisa, 2012.

BRASIL. Agencia Nacional de Vigilância Sanitária. RDC 332 de 01 de Dezembro de 2005. Dispõe sobre a implementação de um sistema de cosmetovigilância, a partir de 31 de dezembro de 2005 em todas as empresas fabricantes e/ou importadoras de produtos de higiene pessoal, cosméticos e perfumes, instaladas no território nacional. Diário Oficial da União, Brasília, 02 dez 2005.

BELOTO, A.; BERTOLINI, G.M.S. Estudo da capacidade funcional ventilatória dos profissionais cabelereiros da cidade de Maringá, estado do Paraná, ano de 2005. Acta Scient Health Sci., v. 28, n. 2, 2008. Disponível em: http://periodicos.uem.br/ojs/index.php/Acta SciHealthSci/article/view/1067/546. Acesso em: 06 ago. 2019.

BREMER, F.M.; HEPP, T. Tratado de cirurgia dermatológica, cosmiatria e laser. [S.I.]: Elsevier, 2012.

CALLAND, R. M. A In: BORELLI, S. Cosmiatria em dermatologia: usos e aplicações. São Paulo: Roca, 2007.

COELHO, M. C. Determinação do ácido fórmico em urina de trabalhadores de uma fábrica produtora de formaldeído. 2009. Monografia - (Especialização) - Faculdade de Farmácia da Universidade do Porto, Cidade do Porto, 2009.

ESTADOS UNIDOS. US Food and Drug Administration, 1997. Centerfor Food Safety and Applied Nutrition. Hair dye products.
FONSECA, M. R. M. Completamente química: Química Geral. 1. ed. São Paulo: FTD, 2001.

FRANQUILINO, E. Cabelos através dos tempos. Revista de Negócios da Indústria da Beleza: edição temática produtos para cabelos, São Paulo, v.4, n.11, p.6-16, ago. 2009.

GASPARINI, F. et al. A simple and green analytical method for the detemination of formaldehyde. J. Braz Chem. Soc., Araraquara, v.19, n.8, p.1531- 1537, 2008. https://doi.org/10.1590/S0103-

$\underline{50532008000800012}$

GOMES, A.L. 0 uso da Tecnologia Cosmética no trabalho do Profissional Cabeleireiro. São Paulo: SENAC, 1999.

GOMES, R. K.; DAMAZIO, M. G. Cosmetologia: descomplicando os princípios ativos. 4. ed. São Paulo: Livraria Médica Paulista , 2013.

HALAL, J. O Crescimento e a estrutura do cabelo. Tricologia e a Química Cosmética Capilar. São Paulo: Cegage Learning, 2012.

HEIMLICH, J.E. Formaldehyde. The Invisible Environment Fact. Sheet Series, The Ohio State University, CDFS-198-08, 2008.

HEIMLICH, J. E. 2008. Formaldeído; O fato do ambiente invisível, Série da folha, a universidade de estado de Ohio, CDFS-19808.

KIRKLAND, D. J. et al. Testing strategies in mutagenicity and genetic toxicology: An appraisal of the guidelines of the European Scientific Committee for Cosmetics and NonFood Products for the evaluation of hair dyes. Genetic Toxicology and Environmental Mutagenesis. v. 588, p. 88-105, 2005. https://doi.org/10.1016/i.mrgentox.2005.09. $\underline{006}$ 
KÖHLER, R. D. A quimica estetica capilar como temática no ensino de química e na capacitação dos profissionais de beleza: estudo de caso. 2011. Dissertação (Mestrado em Educação e Ciências) - Universidade Federal de Santa Maria, Santa Maria, 2011.

LADEIRA, C. A. F. Biomarcadores genotóxicos e polimorfismos genéticos em trabalhadores expostos a formaldeído: estudo de caso. 2009. Dissertação (Mestrado em Biologia Molecular Humana) - Faculdade de Ciências, Universidade de Lisboa, Lisboa, 2009.

LITEPLO, R. G.; BEAUCHAM, R.; MEEK, M. E. Report by International Programme on Chemical Safety IPCS. Canadian Centre for Occupational Health and Safety (CCOHS), 2002.

LORENZINI S. Efeitos adversos da exposição ao formaldeído em cabeleireiros. 2012. Tese (Doutorado)- Faculdade de Medicina, Universidade Federal do Rio Grande do Sul, Rio Grande do Sul, 2012.

MACAGNAN, K.; SARTORI, M. R. K.; CASTRO, F. G. Sinais e sintomas da toxicidade do formaldeído em usuários de produtos alisantes capilares. Caderno da Escola de Saúde, Curitiba, v.1, n.4, ago./dez., 2010.

MANSUR, C.; GAMONAL, A. Cabelo normal. In: KEDE, M. P. V; SABATOVICH, O. Dermatologia estética. São Paulo: Atheneu, 2004.

NIOSH - National Institute for Occupational Safety and Health. Manual of Analytical Methods (NMAN): method 3500 (2)formaldehyde, 4.ed. US Department Health, Education and Welfare, 1994

NOHYNEK, G. J. et al. Toxicity and human health risk of hair dyes. Food and Chemical Toxicology., Amsterdã., v. 42. p. 517-543, 2004.

https://doi.org/10.1016/i.fct.2003.11.003
NORBACK, D. et al. Asthmatic symptoms and volatile organic compunds, formaldehyde, and carbon dioxide in dwellings. Occup Environ Med., v. 52, n. 6, jun., 1995. Disponível em: $<$ https://www.ncbi.nlm.nih.gov/pubmed/762 7316\#>. Acesso em: 06 ago. 2019. https://doi.org/10.1136/oem.52.6.388

OLIVEIRA, V. G. Cabelos: uma Contextualização no Ensino de Química. Unicamp, São Paulo,2013.

OLIVEIRA, R. A. G. et al. A química e toxidades dos corantes de cabelo. Quimica Nova, v. 37, n. 6, maio 2014. https://doi.org/10.5935/0104$\underline{8899.20150047}$

PERREIRA, J. M. Propedêutica das doenças dos cabelos e do couro cabeludo. 3. ed. São Paulo: Atheneu, 2001

PINHEIRO, A. O formol no processo de alisamento dos cabelos. Cosmetics \& Toiletries, São Paulo, v.16, p. 40, 2004.

PINHEIRO, A.KOSMOCIENCE - A arte de colorir os cabelos. Centro de Pesquisa e desenvolvimento.

REIS, M. Completamente Química: Química Orgânica. 1. ed. São Paulo: FDT, 2001.

TOUTIANOUSH, A.; SCHNEPF, J.; ELHASHANI, A.; TIEKE, B. Selective lon trasport and complexation in layer-by-layer Assemblies of p-Sulfonato- caixa[n]arenes and Cationic Polyelectrolytes. Adv Functn Mater. v. 15, n. 4, p. 700-8, 2015. https://doi.org/10.1002/adfm.200400223

WEKEFIELD, J. C. Formaldehyde general information. Report by Health Protection 